\title{
Brain and Body Hyperthermia Associated with Heroin Self-Administration in Rats
}

\author{
Eugene A. Kiyatkin and Roy A. Wise \\ Behavioral Neuroscience Branch, National Institute on Drug Abuse-Intramural Research Program, \\ Baltimore, Maryland 21224
}

\begin{abstract}
Intravenous heroin self-administration in trained rats was accompanied by robust brain hyperthermia $\left(+2.0-2.5^{\circ} \mathrm{C}\right)$; parallel changes were found in the dorsal and ventral striatum, mediodorsal thalamus, and deep temporal muscle. Temperature began to increase at variable latency after a signal of drug availability, increased reliably $\left(\sim 0.4^{\circ} \mathrm{C}\right)$ before the first lever press for heroin, increased further $\left(\sim 1.2^{\circ} \mathrm{C}\right)$ after the first heroin injection, and rose more slowly after the second and third injections to stabilize at an elevated plateau $\left(39-40^{\circ} \mathrm{C}\right)$ for the remainder of the session. Brain and body temperature declined slowly when drug self-administration was terminated; naloxone precipitated a much more rapid decrease to baseline levels. Changes in temperature were similar across repeated daily sessions, except for the increase associated with the first selfadministration of each session, which had progressively shorter latency and greater acceleration. Despite consistent biphasic
\end{abstract}

fluctuations in movement activity associated with heroin selfadministrations (gradual increase preceding the lever press, followed by an abrupt hypodynamia after drug infusion), mean brain temperature was very stable at an elevated plateau. Only mean muscle temperature showed evidence of biphasic fluctuations $\left( \pm 0.2^{\circ} \mathrm{C}\right)$ that were time locked to and correlated with lever pressing and associated movements. Drug- and behaviorrelated changes in brain temperature thus appear to reflect some form of neuronal activation, and, because temperature is a factor capable of affecting numerous neural functions, it may be an important variable in the control of behavior by drugs of abuse.

Key words: brain temperature; opiates; heroin; neural activation; drug-taking behavior; thermorecording in behaving animals
Although it is generally assumed that brain temperature is a strictly regulated homeostatic variable with a range of fluctuations more restricted than those of body temperature (Satinoff, 1978; Bullock et al., 2001), relatively large increases in brain temperature $\left(1.0-2.0^{\circ} \mathrm{C}\right)$ have been found in animals exposed to various biologically significant stimuli or engaged in different behaviors. Temperature in different brain structures increases during exploration of new environment, treadmill running and swimming (Moser et al., 1993), feeding (Abrams and Hammel, 1964), and handling by an experimenter (Delgado and Hanai, 1966). Brain temperature also significantly differs between day and night, phasically rising during movement episodes (i.e., drinking, feeding, and running) and falling during sleep (Abrams and Hammel, 1965); it shows significant correlation with EEG during transition between sleep and wakefulness, as well as after environmental stimulation (Delgado and Hanai, 1966). Because many variables underlying neuronal excitability [i.e., membrane potential (Thompson et al., 1985), transport via ion-selective channels (Rosen, 2001), and amplitude and duration of single-unit spikes (Thompson et al., 1985; Erickson et al., 1996)] are temperature dependent, changes in brain temperature occurring under behavioral conditions may be an important factor in central regulation of various neural functions, including adaptive behavior.

\footnotetext{
Received Aug. 20, 2001; revised Oct. 15, 2001; accepted Nov. 8, 2001.

We thank P. Leon Brown for assistance in data analysis and suggestions on this manuscript.

Correspondence should be addressed to Eugene A. Kiyatkin, Behavioral Neuroscience Branch, National Institute on Drug Abuse-Intramural Research Program, 5500 Nathan Shock Drive, Baltimore, Maryland 21224. E-mail: ekiyatkin@intra. nida.nih.gov

Copyright (C) 2002 Society for Neuroscience $\quad 0270-6474 / 02 / 221072-09 \$ 15.00 / 0$
}

To explore to what extent brain temperature changes during drug-taking behavior, temperature was monitored in several brain structures and body during the development and maintenance of intravenous heroin self-administration in rats. Three limbic structures implicated in goal-directed behavior, the dorsal striatum (caudate putamen), ventral striatum [nucleus accumbens (NAcc)], and mediodorsal thalamus (MDT), were chosen as brain targets for recording. Deep temporal muscle, a head muscle that has a blood supply similar to that of the brain, was chosen as a site for assessing body temperature. Measurements were taken with high temporal resolution throughout several daily sessions from the first accidental drug-reinforced lever press in drug-naive animals to the stable, highly cyclical lever-pressing behavior in trained animals.

\section{MATERIALS AND METHODS}

Animals and surgery. Eight male Long-Evans rats, weighing 400-500 $\mathrm{gm}$ and received from Charles River Laboratories (Greensboro, NC), were used. Each rat was housed individually (12 hr light/dark cycle, beginning at 7:00 A.M.) with access to food and water ad libitum. All protocols were performed in compliance with the NIH Guide for the Care and Use of Laboratory Animals (National Institutes of Health, Publication 865-23) and were approved by the Animal Care and Use Committee of the National Institute on Drug Abuse-Intramural Research Program.

Each rat was anesthetized with a mixture of ketamine $\mathrm{HCl}(80 \mathrm{mg} / \mathrm{kg}$, i.m.) and xylazine $(10 \mathrm{mg} / \mathrm{kg}$, i.m. $)$ and mounted in a stereotaxic apparatus. Holes were drilled through the skull over three areas of interest: the right dorsal striatum $(1.4 \mathrm{~mm}$ anterior and $2.0 \mathrm{~mm}$ lateral of bregma), the left NAcc shell (1.4 mm anterior and $0.9 \mathrm{~mm}$ lateral of bregma), and the right MDT (2.8 $\mathrm{mm}$ posterior and $1.5 \mathrm{~mm}$ lateral of bregma). The dura matter was carefully retracted, and each temperature probe was slowly lowered to the area of interest. A fourth temperature probe was implanted in deep temporal muscle (musculus temporalis), and the four probes were secured 
with dental cement to three stainless steel screws threaded into the skull. During the same operation, each animal was implanted with an intravenous jugular catheter with an exit that was fixed to the head assembly. Experimentation began after a $3 \mathrm{~d}$ recovery period and continued for the next 6-10 daily sessions.

Temperature probes and recording instruments. Thermocouple probes were prepared from insulated copper and constantin (a 60\% copper, $40 \%$ tin alloy) wires (TW-35P; diameter of $\sim 125 \mu \mathrm{m}$ ) obtained from Physitemp Instruments (Clifton, $\mathrm{NJ}$ ). Thermocouple probes were made by mechanically removing the insulation $(\sim 200-400 \mu \mathrm{m})$ from the tip of each wire, soldering the tips together, and reinsulating with polyester microshrink tubing and epoxy. The wires were connected to copper and constantin pins fixed in a plastic connector with epoxy. All probes were tested at the same environmental temperature, and only those that showed temperature deviations within $0.1^{\circ} \mathrm{C}$ were used for implantation. During experiments, the probes were connected to the recording instrument (a 16-channel thermocouple data acquisition system Thermes-16; Physitemp Instruments) via individual sockets, a common cord, and a nine-channel electric swivel. All connecting materials for temperature recordings were prepared from either copper or constantin to maintain the temperature gradient established at the initial interface of the two metals. The intravenous catheter was connected to a syringe pump via a polyethylene catheter fed through a swivel commutator for the recording leads.

During the session, temperatures were continuously recorded and stored in computer memory at $2 \mathrm{sec}$ bins together with event marks to indicate the times of noted behavioral observations. Temperature in the room was maintained automatically at $23^{\circ} \mathrm{C}$, and temperature in the experimental chamber $\left(23-24^{\circ} \mathrm{C}\right)$ was also continuously recorded.

Experimental protocol. All recordings took place during the day phase of the animal's cycle (10:00 A.M. to 6:00 P.M.) in an electrically shielded Plexiglas chamber $(35 \times 35 \times 40 \mathrm{~cm})$ equipped with an operant lever $(6$ $\mathrm{cm}$ above the floor surface); a speaker and a small light source were mounted above the lever. Each day, the rats were brought from their housing facility, placed in the chamber, connected to the recording instrument and syringe pump, and allowed to habituate to the experimental environment, during which the lever was covered with a transparent cover. During this period, rats were engaged in intense exploration of the cage (locomotion, grooming, and rearing) that was associated with temperature increase. After these behavioral changes disappeared and temperature stabilized (60-90 $\mathrm{min})$, each rat was exposed to a compound sensory stimulus ( $18 \mathrm{sec}$ tone plus $18 \mathrm{sec}$ light), after which the cover was removed and the lever became accessible. This compound stimulus was presented when the rat was in quiet resting or sleep-like conditions with no overt movements. Each voluntary lever press resulted in heroin inf usion $(100 \mu \mathrm{g} / \mathrm{kg}$ delivered in a volume of $180 \mu \mathrm{l}$ over a 18 sec period), which was accompanied by the same compound light and sound stimulus. Each heroin injection resulted from voluntary lever presses; experimenter-initiated injections were never used. Drug selfadministration was continued for $5 \mathrm{hr}$, after which the lever was restricted by the transparent cover again, and temperature recordings were continued for 1 additional hour. This basic protocol was maintained for at least five daily sessions. In some animals, single lever presses within a session were reinforced by heroin at double the usual dose $(200 \mu \mathrm{g} / \mathrm{kg}$ over a 36 sec period). Such double-dose self-administrations were made in well trained rats (see criteria in Results), and they never occurred more than once in a session. In some rats, we also tested the effect of naloxone (1 $\mathrm{mg} / \mathrm{kg}$ ), which was injected subcutaneously during the final session after the standard $5 \mathrm{~d}$ protocol (on days 6,7 , or 8 ) session. These injections were made $\sim 3-4 \mathrm{hr}$ after the beginning of drug taking. Before the first drug session, each rat was habituated to the testing environment for two daily sessions (4-6 hr each), during which it was exposed to light and sound stimulus.

Histology. After completion of the experiments, each rat was deeply anesthetized and perfused with a $10 \%$ formalin solution. The brain was removed and stored for subsequent histological processing. The location of the recording sites was determined from 50-100 $\mu \mathrm{m}$ slices mounted on glass slides and stained with cresyl violet.

Data analysis. Our analysis was based on determining the pattern of temperature changes proceeding and after critical events of drug-taking behavior. These events were as follows: (1) presentation of light-tone compound stimulus that was used to signal the start of each session; (2) the first lever press and heroin injection of each session; (3) each subsequent self-administration of a session; (4) lever presses reinforced by heroin at the double dose; (5) the last self-administration of a session;

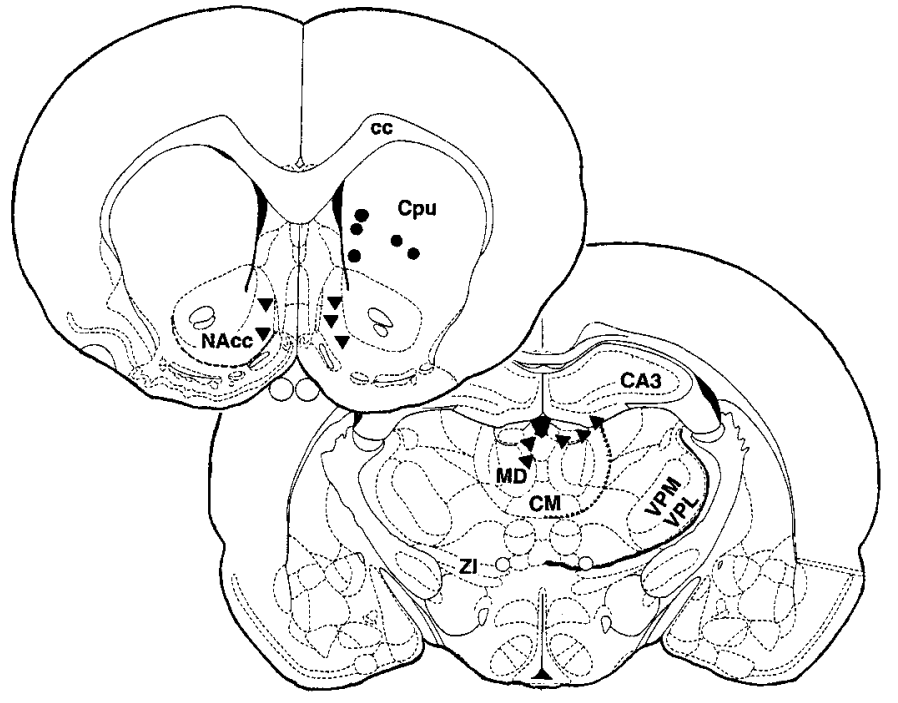

Figure 1. Coronal sections of the brain illustrating reconstructed locations of thermocouple tips in rats used in the present study. $c c$, Corpus collosum; $C p u$, caudate putamen; NAcc, nucleus accumbens; $C A 3$, field CA3 of Ammon's horn; $Z I$, zona incerta; $M D$, mediodorsal thalamic nucleus; $C M$, central medial thalamic nucleus; $V P M$, ventroposterior thalamic nucleus, medial; $V P L$, ventroposterior thalamic nucleus, lateral. Dotted lines show ventral border of NAcc (anteroposterior, +1.20), dorsomedial thalamus, and thalamus (anteroposterior, -2.80). Atlas of Paxinos and Watson (1986) was used to prepare drawings.

and (6) the administration of naloxone. Although changes in temperature were calculated for each behavioral event during each session, most analyses were made on trained rats, defined as rats that showed regular and sustained lever pressing throughout full sessions (for details, see Results). Significance of temperature differences was evaluated using an ANOVA with repeated measures, followed by Scheffé tests at $1 \mathrm{~min}$ intervals. The data were presented as changes in absolute temperature, changes relative to the pre-event baseline (baseline is 0), rates of change, and temperature differences between recording sites.

\section{RESULTS}

Data were analyzed from five of the eight rats. Three animals were excluded from the final analysis because of problems with either thermocouple connections or intravenous catheters. In each of the five rats, histological examination confirmed that thermocouple probes were localized within the intended target (Fig. 1). The probes aimed at NAcc were located in medial areas of the structure (shell), and probes aimed at MDT were located in the upper part of mediodorsal thalamic complex (mediodorsal, lateral habenular, and paraventricular thalamic nuclei), with one electrode located in the border between MDT and hippocampus. In each of these animals, temperature recordings with heroin access were continued for at least five daily sessions; a total of 32 sessions were analyzed.

\section{Heroin self-administration}

After the first exploratory lever press reinforced by heroin on day 1 , each of the five analyzed rats continued lever pressing during this and all subsequent sessions (Fig. $2 A$ ). Whereas the intervals between self-administrations varied from 10 to $61 \mathrm{~min}(37.13 \pm$ $24.59 \mathrm{~min}$; mean $\pm \mathrm{SD})$ on day 1 , the mean inter-injection intervals became progressively shorter on subsequent days (20-24 min during the two final sessions) as lever pressing became more regularly spaced. Drug-taking behavior was accompanied by cyclic fluctuations in movement activity with gradual increases in locomotion, grooming, rearing, and circling before each lever 

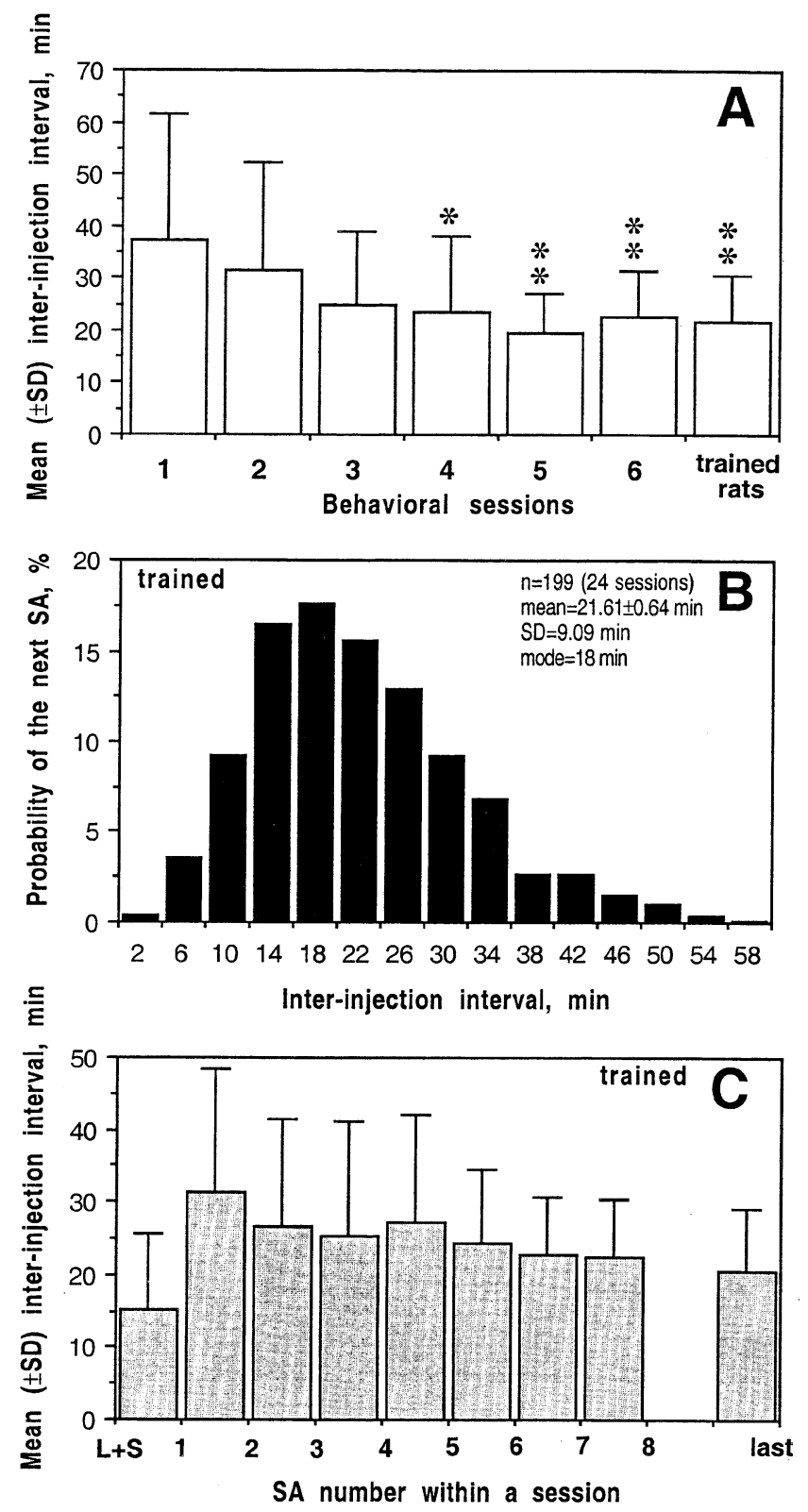

Figure 2. $A$, Mean \pm SD inter-injection interval as a function of session number. $B$, Probability of responding as a function of inter-injection interval ( $n=199$ from 24 sessions). $C$, Mean \pm SD latency to the next lever press as a function of self-administration number. $L+S$ indicates time interval between presentation of light-tone cue and the first heroin self-administration of a session.

press and abrupt transient cessation of activity (freezing) after each heroin infusion. Although evident in each session, the strength and duration of freezing became progressively weaker during repeated sessions, whereas hyperactivity progressively increased with the eventual appearance of stereotypic head movements and chewing.

Animals were designated as "trained' based on regularity of lever pressing and stability of inter-injection intervals over the 5 hr of a session. Three animals were considered trained on day 2 and two more animals on day 3. In these trained rats, the distribution of inter-injection intervals ( $n=199$ from 24 sessions) was slightly skewed (Fig. $2 B$ ). After each lever press, the probability of the next lever press was close to zero for the first 4 min, then gradually increased, peaking at 16-20 min, and then dropped again to low levels $(<2 \%)$ from 36 to $60 \mathrm{~min}$ after the selfinjection. The first inter-injection interval was the longest and most variable (range, 9-73 min; mean, $31.38 \pm 3.51 \mathrm{~min}$; SD, $17.17 \mathrm{~min}$ ); all subsequent intervals were shorter and, on average, very stable throughout the session (Fig. 2C). With each subsequent self-administration, the variability of inter-injection intervals gradually decreased (see almost twofold decrease in SD), suggesting progressively tighter regulation. Trained rats did not immediately press the lever after presentation of the drug-related cue; the latency to the first-in-session lever press varied from 3 to $33 \mathrm{~min}$ (mean of $15.28 \pm 2.05 \mathrm{~min}$ ), with no significant differences across sessions.

\section{Tonic temperature changes during heroin self-administration session}

In trained animals, heroin self-administration was accompanied by a robust temperature increase in all recording sites (Fig. 3). Temperatures increased slightly but significantly after presentation of the heroin-related cue $\left(\sim 0.3-0.5^{\circ} \mathrm{C}\right)$ and before the first lever press. Temperatures increased more strongly $\left(1.6-1.8^{\circ} \mathrm{C}\right)$ after the first heroin self-administration of a session. They continued to increase, although at progressively decreasing rates, after the second and the third self-administrations, before stabilizing at high $\left(2.2-2.5^{\circ} \mathrm{C}\right.$ above baseline) but relatively stable plateaus. Although similar fluctuations were evident in records from each site, basal temperatures in the NAcc were significantly higher $\left(\sim 0.4^{\circ} \mathrm{C}\right)$ (Fig. $\left.3 A\right)$ than those in other brain sites and in the muscle. Temperatures in the dorsal striatum and MDT were similar to those in the muscle. Temperature increased significantly more in all brain sites than in the muscle after presentation of the heroin-related cue; relative changes in the brain sites were quite similar to one another (Fig. $3 B, C$ ). The brain-muscle temperature differential was maximal $\left(\sim 0.25^{\circ} \mathrm{C}\right)$ immediately before the first self-administration; this differential gradually decreased during the remainder of the session.

The pattern of tonic temperature increase was typical of each animal and each session, despite between-animal differences in the rate of heroin self-administration and in the absolute values of temperature plateaus (Fig. 3, right panel). This pattern was quickly developed during the initial sessions, although changes in temperature and inter-injection intervals were more variable in initial sessions (Fig. 3, 9-1). Because tonic changes in brain temperature occurring during heroin self-administration session were more tightly correlated with each other than with changes in muscle temperature, further analysis was performed for two recording sites: NAcc and temporal muscle.

\section{Phasic temperature changes associated with individual behavioral events}

In drug-naive rats, the compound light-tone stimulus, which was subsequently to be associated with each heroin selfadministration, caused no obvious behavioral changes but a shortterm increase in temperature that was consistently greater in the NAcc than in the muscle (Fig. 4, top row). NAcc and muscle temperatures also gradually increased again when the rats initiated searching behavior that eventually resulted in the first ever lever press. This increase was greater $\left(\sim 0.5^{\circ} \mathrm{C}\right)$ and longer lasting, it peaked $\left(\sim 38.2^{\circ} \mathrm{C}\right.$ for NAcc) at the moment of the lever press, and it was stronger in the brain than in the muscle.

In trained animals, presentation of the same light plus tone stimulus (now a heroin-related cue) also failed to result in con- 

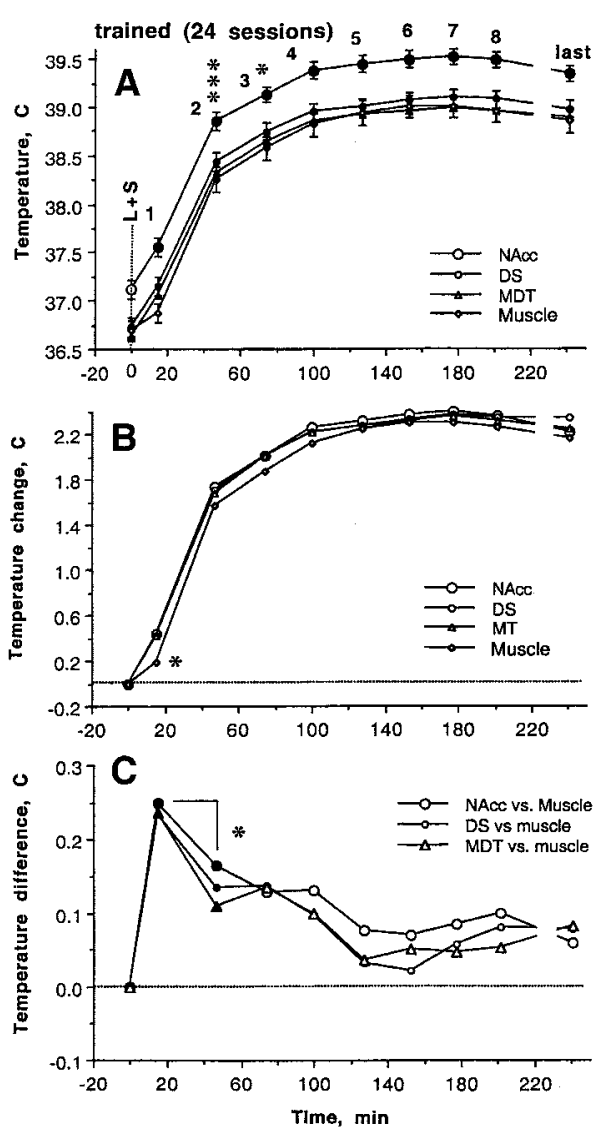

sistent changes in behavior. All rats, however, with highly variable latencies, initiated searching behavior (locomotion, grooming, washing, etc.) that finally resulted in the first lever press of the day. Although temperature increased significantly from the second minute after the cue stimulation and maintained at relatively stable levels $\left(\sim 0.2^{\circ} \mathrm{C}\right)$ for the next 8 min (Fig. 4, bottom row), it was impossible to accurately evaluate statistical significance of this effect because, in 7 of 22 sessions, rats performed the first lever press within this period. The postcue increase in NAcc temperature was significant $\left(F_{(14,164)}=5.09 ; p<0.001\right.$; from 4 to $10 \mathrm{~min}$ ), even when only 15 cases with no lever press within 10 min were analyzed. More consistent changes in temperature were found when data were analyzed with respect to the first heroin self-injection (right). In this case, NAcc temperature gradually increased for the $10 \mathrm{~min}$ preceding the first lever press $\left(F_{(9,259)}=\right.$ 17.27; $p<0.0001$; Scheffé $F$ test). Although muscle temperature also significantly increased before the first lever press, this change was much weaker $\left(F_{(9,259)}=3.58 ; p<0.01\right)$. In trained rats, both basal and peak temperatures immediately before the first lever press were significantly lower than in drug-naive rats.

After the first self-administration of a session in trained rats, temperature continued to increase by over $1^{\circ} \mathrm{C}$, with similar changes in NAcc and muscle (Fig. 5A). The increase was evident during the period of hypoactivity after heroin injection $(\sim 0.5-8$ min) and continued as the rat became active preceding the second lever press. The time course of temperature increase was dependent on the animal's experience. On day 1 (drug-naive state), an increase in NAcc temperature occurred with $\sim 7$ min latency; latency became progressively shorter on subsequent days (Fig. $5 B)$. Similar changes were typical of muscle temperature. Although mean magnitude of temperature increase for the first 10
Figure 3. Mean changes in temperature (left) and sample records from individual animals (right) as a function of time during heroin self-administration session in trained rats. $A$, Absolute temperatures in different recording sites. Filled symbols show values significantly higher than those seen immediately before the light and sound stimulus. Asterisks indicate significance with respect to the previous point $\left({ }^{*} p<0.05\right.$; $* * * p<$ $0.001)$. $B$, Temperature changes at different recording sites (with respect to baseline values immediately before presentation of the light-sound stimulus baseline is 0 ) as a function of time within session. Asterisk indicates that the initial changes in temperature in all brain sites was significantly $(p<0.05)$ greater than that in the muscle. $C$, Brain-muscle temperature difference as a function of time within session. Filled symbols show significance with respect to baseline, and asterisk indicates significant decrease in the parameter after the first heroin self-injection. Original records show changes in temperature at each of the four recording sites, with vertical lines indicating the times of each lever press. $L+S$ indicates presentation of heroin cue. 1 indicates the first lever press of a session. The number at the last vertical line shows the number of the last self-administration of a session. Double numbers above the graphs depict the number of rat and session, respectively. period (Fig. 5C), the absolute values of NAcc and muscle temperature by the moment of the second lever press were not different in different sessions. Temperature changes associated with the second self-administration of a session resembled those of the first self-administration, but the postdrug increases were more delayed and much smaller in magnitude. The pre-lever press temperature increase completely disappeared, and the postinjection increase was minimal after the third selfadministration. In both cases, temperature changes in the muscle were stronger than in the NAcc.

In contrast to the initial three self-administrations of each session, which were associated with temperature increases, brain temperature remained very stable with no phasic fluctuations within the cycle in association with each subsequent heroin selfadministration. Although NAcc temperature fluctuated considerably around individual self-administrations in different rats and sessions (Fig. 6, right panel), an ANOVA with repeated measures revealed no reliable effect of time $\left(F_{(19,2499)}=0.47 ; p=0.55\right)$ on temperature across rats and sessions [all self-injections except the first three, the last one, and those with atypically short ( $<10 \mathrm{~min})$ or long (>30 min) inter-injection intervals were averaged for this analysis]. A very weak but significant biphasic effect of time, however, was found for changes in muscle temperature, which slightly increased before the lever press, abruptly decreased at 2-4 min after heroin infusion, and slowly increased again preceding the next self-administration. When the dose of heroin was unexpectedly doubled, the next lever press was delayed significantly $(28.23 \pm 3.08$ vs $18.69 \pm 1.81 \mathrm{~min}$ for the single-dose injection; $p<0.05$; Student's $t$ test), but the changes in NAcc and muscle temperature were surprisingly similar after the two types 
Figure 4. Changes in NAcc and muscle temperature after presentation of the compound light-sound stimulus (left) and before the first self-administration of a session (right) in drugnaive (top) and trained (bottom) rats. Arrows indicate the moments of cue presentation and the first lever press $(S A)$, respectively. Filled symbols show significant changes relative to the last precue or the last prepress value. In the drug-naive state, numbers of cases for lighttone stimulus and the first lever press were 10 ( 2 presentations in each rat) and five, respectively. In the drug-trained state, 22 cases were averaged for each event. Because in seven cases lever presses occurred within $10 \mathrm{~min}$ after the light-tone cue, mean values from the fourth minute were obtained with progressively smaller numbers of cases per group (from 21 at $4 \mathrm{~min}$ to 15 at $10 \mathrm{~min}$ ).
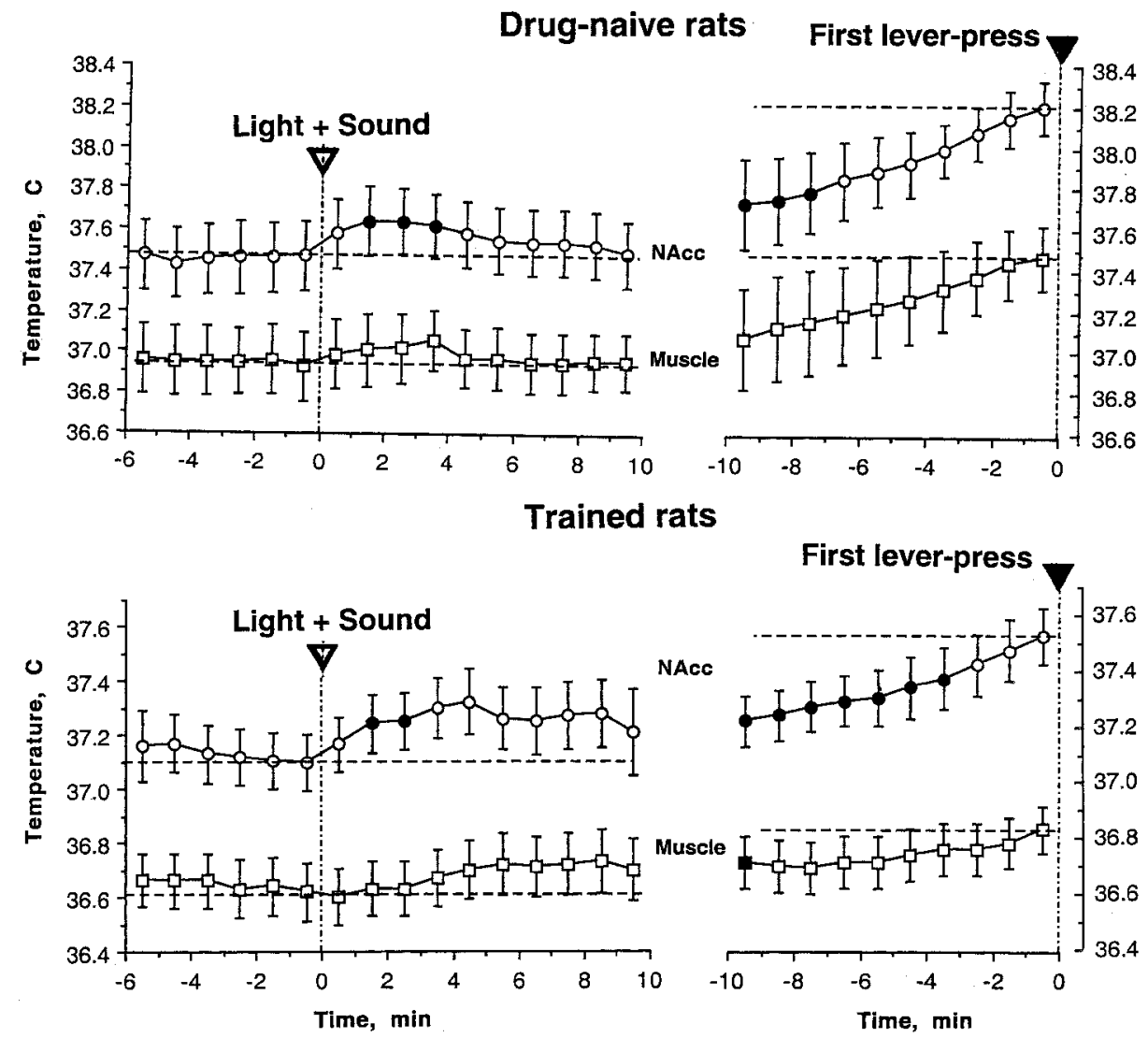

of injection. In the double-dose condition, the only difference was a slight exaggeration of the postinjection decrease in muscle temperature.

In three rats (four later sessions), we tested the effect of naloxone $(1 \mathrm{mg} / \mathrm{kg}$, s.c.) injected to heroin self-administering rats at the later part of the session when tonic temperature was locked at the elevated plateau. Naloxone induced an abrupt (20 min) and strong return of brain and muscle temperature to baseline levels (Fig. 7). Naloxone affected body temperature before it affected brain temperature (latency to significant decrease was 3 vs $5 \mathrm{~min}$ ). In all cases, no heroin self-injections occurred for at least $1 \mathrm{hr}$ after naloxone administration. Temperature also decreased after the last heroin self-administration when the operant lever was closed (see original examples in Fig. 3). In this case, however, the temperature was stable for some time after the last injection (Fig. 7 , control) and return to baseline occurred at $\sim 90-120 \mathrm{~min}$. The return to baseline after naloxone was at least four times faster than that occurring after the last drug self-administration.

\section{DISCUSSION}

In light of the traditional belief that brain temperature is defended within narrow limits, robust brain hyperthermia associated with heroin self-administration may seem surprising. Mean NAcc temperature during the maintenance phase of drug-taking behavior in trained rats was $\sim 2.5^{\circ} \mathrm{C}$ above the resting baseline, with increases often exceeding $3^{\circ} \mathrm{C}$ (to $40-$ $40.5^{\circ} \mathrm{C}$ ) in individual animals. Such extreme hyperthermia looks also surprising from a pharmacological standpoint because the dose of heroin in our experiments $(0.1 \mathrm{mg} / \mathrm{kg})$ was much lower than the traditional low doses of opiate drugs causing hyperthermia (for review, see Adler and Geller, 1993).
The magnitude of brain and muscle hyperthermia observed after the first-in-session heroin self-injection, for example, was comparable or even higher with that observed in the body of intact animals passively administered with this or similar drugs (i.e., morphine) at doses 10 - to 40-fold higher. This underscores the fact that the functional state of an animal, as well as the specific combination of dose, route, and context of drug administration, are important determinants of an organism's response to opiate drugs. This may be especially true with respect to brain temperature, which shows relatively strong increases $\left(1-2^{\circ} \mathrm{C}\right)$ after a variety of mild arousing stimuli, such as the testing environment, tail-pinch, and interaction with another animal of the same or opposite sex (Brown et al., 2001; Kiyatkin and Wise, 2001).

\section{Pharmacological and other influences as a cause of brain hyperthermia during heroin self-administration}

The effects of heroin on temperature were dependent on the amount of previous drug experience and the level of preinjection temperature. Whereas the first heroin injection of a session was associated with rapid and profound temperature increase, smaller increases were seen after the next one or two injections, and no additional increase was seen after subsequent injections. Thus, there appears to be an upper limit of heroin-induced hyperthermia. After reaching this limit after the first three heroin injections, the temperature was tightly "maintained" at these increased levels by subsequent repeated drug self-administration, and it remained stable even when dose was unexpectedly doubled. This stability of brain temperature was unexpected in light of profound biphasic fluctuations in movement activity, arterial blood pressure (Kiyatkin and Stein, 1993), and impulse activity of ventral teg- 

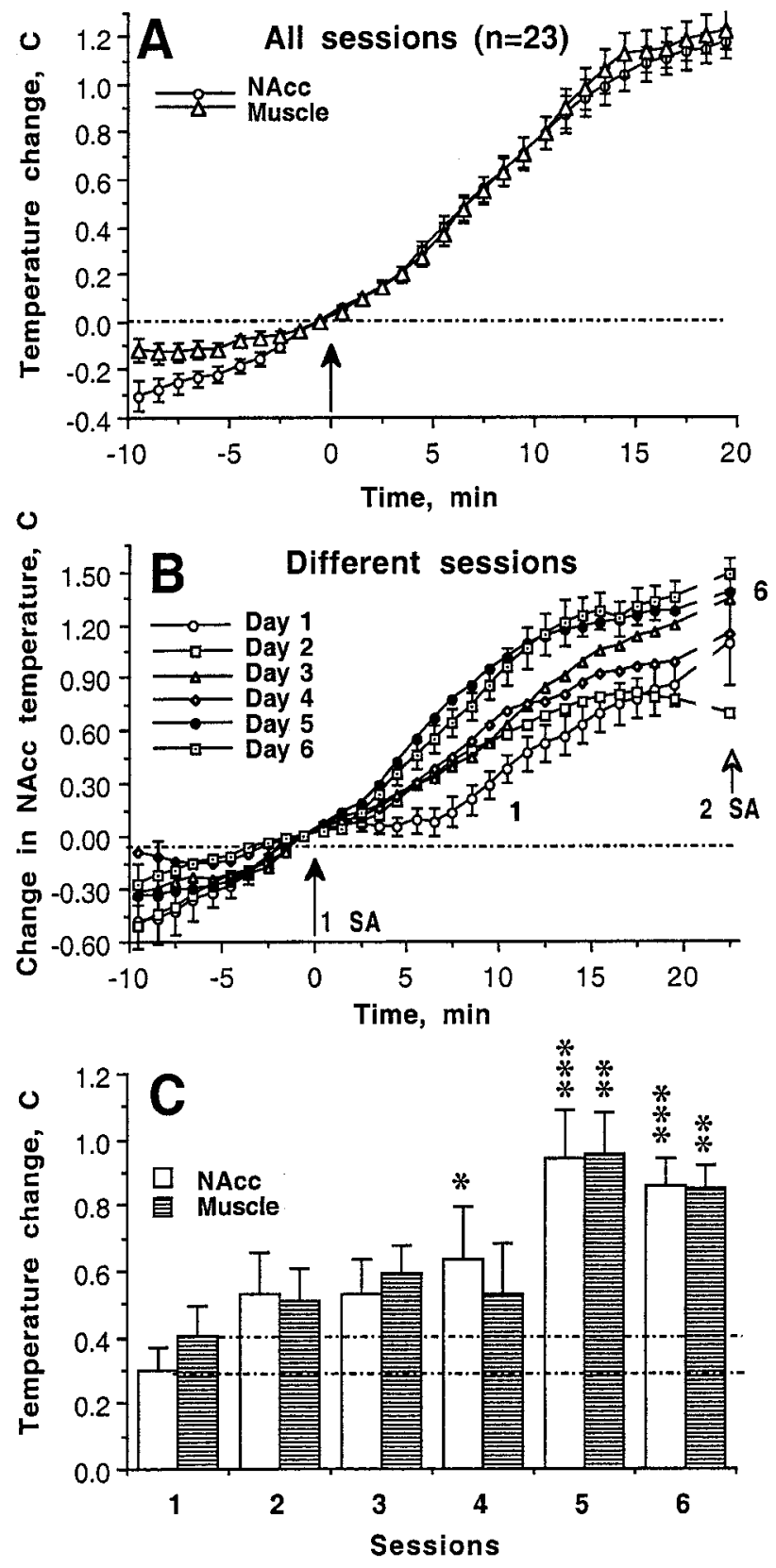

Figure 5. Changes in NAcc and muscle temperature associated with the first heroin self-administrations of a session. $A$ shows mean \pm SEM temperature changes before and after the first self-administrations in trained animals (23 sessions). Each value from the second to the last minute is significantly higher than the last preinjection value, with no significant differences between NAcc and muscle (NAcc, $F_{(19,479)}=91.89$; muscle, $F_{(19,479)}=58.26$; effect of time is highly significant in each case, $p<0.0001$; Scheffé $F$ test). $B$ shows temperature changes associated with the first-in-session heroin self-administration on days $1,2,3,4,5$, and 6 . The last values correspond to the moment immediately before the second lever press $(2 S A)$. SEs are shown only for days 1 and 6 . The times of initial self-administration are indicated by vertical arrows; basal values are shown as horizontal dotted lines. $C$ shows mean changes in NAcc and muscle temperature at $10 \mathrm{~min}$ after the first-in-session heroin selfadministration in consecutive sessions. Asterisks show significant increases relative to day $1\left({ }^{*} p<0.05\right.$; ** $p<0.01$; ** $p<0.001$; Student's $t$ test $)$.

mental area (VTA) neurons (Kiyatkin and Rebec, 2001) associated with individual heroin self-administrations. It seems likely, therefore, that self-injected heroin intake is sufficient to maintain systemic opiate levels at or above their maximum effective concentration with respect to thermoregulatory challenge.

Although the pharmacological actions of heroin appear to be the major causes of brain hyperthermia occurring in our experiments, changes in neural activity associated with arousal and behavioral activation make significant contributions to this phenomenon in the initial phase of drug-taking behavior. In trained animals, brain temperature reliably increased after presentation of the light-tone cue before the first drug self-administration; this increase was always time locked to the prepressing behavioral activation but was not invariably time locked to the cue itself. Both the postcue and the subsequent pre-lever press increases in temperature were more pronounced in all brain sites than in the muscle, suggesting some form of neural activation as their primary mechanism. Surprisingly, the heroin-related cue did not come to elicit immediate drug seeking within the few days of training in the present study, and it never came to induce larger temperature increases than were seen on the first day, when the animals were in the drug-naive state.

Despite the fact that they did not increase in magnitude, the elevations in brain temperature seen in trained rats before the first-in-session lever press may reflect "anticipatory" brain activation resulting from the conditioned association of the cue and lever availability with previous heroin injections. The increase in brain temperature after the compound light-tone stimulus was, on the first day, attributable only to stimulus novelty; it had no conditioned significance for the animal at that time. However, brain temperature response to such stimuli habituates in 4-5 d if they are simply repeated without other associations (Brown et al., 2001). The fact that the strength of the effect did not decrease on subsequent days suggests that the temperature change was a correlate of something akin to Pavlov's "investigatory reflex," a response common to novel stimuli, unconditioned stimuli, and conditioned stimuli. Such a response to a novel stimulus would not be sustained if the stimulus were not given signal significance by association with reinforcer (Pavlov, 1927). Recent discussions of the growth of incentive motivation (Robinson and Berridge, 2001) and reward anticipation (Schultz, 1997; Schultz and Dickinson, 2000) might lead one to expect much stronger effects on the arousal response and the related temperature increases as the cue became associated with heroin infusions. It remains possible that the cue in the present study would have acquired stronger associative strength with additional pairings. A light-tone stimulus paired with self-administered cocaine has been shown capable of reinstating lever pressing in animals that have had their habit extinguished (Meil and See, 1996; Grimm et al., 2001). However, light and tone stimuli are minimally effective in reinstating responding when compared with priming injections of the drug itself (de Wit and Stewart, 1981, 1983). Thus, it is possible that lights and tones are not stimuli that rats are particularly responsive to. In any case, our data show, consistent with Pavlov's (1927) teachings, that the initial response to the cue merely failed to undergo normal habituation, not that is increased with training.

\section{Mechanisms underlying brain hyperthermia during heroin self-administration}

Although the central actions of heroin would seem to be a major factor in the robust brain hyperthermia found in our experiments, the mechanisms underlying this hyperthermia are not clear. It is known that hyperthermia induced by morphine at low doses is accompanied by an increase in whole-body oxygen consumption and heat production (Lynch et al., 1987, 1990), parameters that 


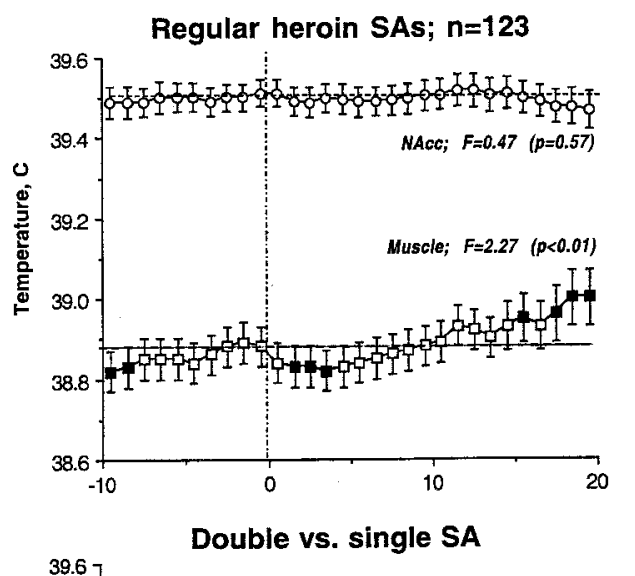

Figure 6. Mean \pm SEM (left) and individual (right) changes in temperature associated with consecutive single- (left, top) and double-dose (left, bottom) heroin self-administrations $(S A)$ during the maintenance phase of drug-taking behavior. Filled symbols indicate values significantly lower or higher than the last preinjection value. Original records show changes in temperature in each of the four recording sites, and vertical lines indicate the moments of lever presses and their numbers. Double numbers above the graphs indicate the number of rat and session. The first mean graph represents an average of 123 heroin self-injections (5 rats, 23 sessions), and the second mean graph represents averages of 23 double-dose selfinjections, which were compared with 23 control single-dose injections either before or after this event.
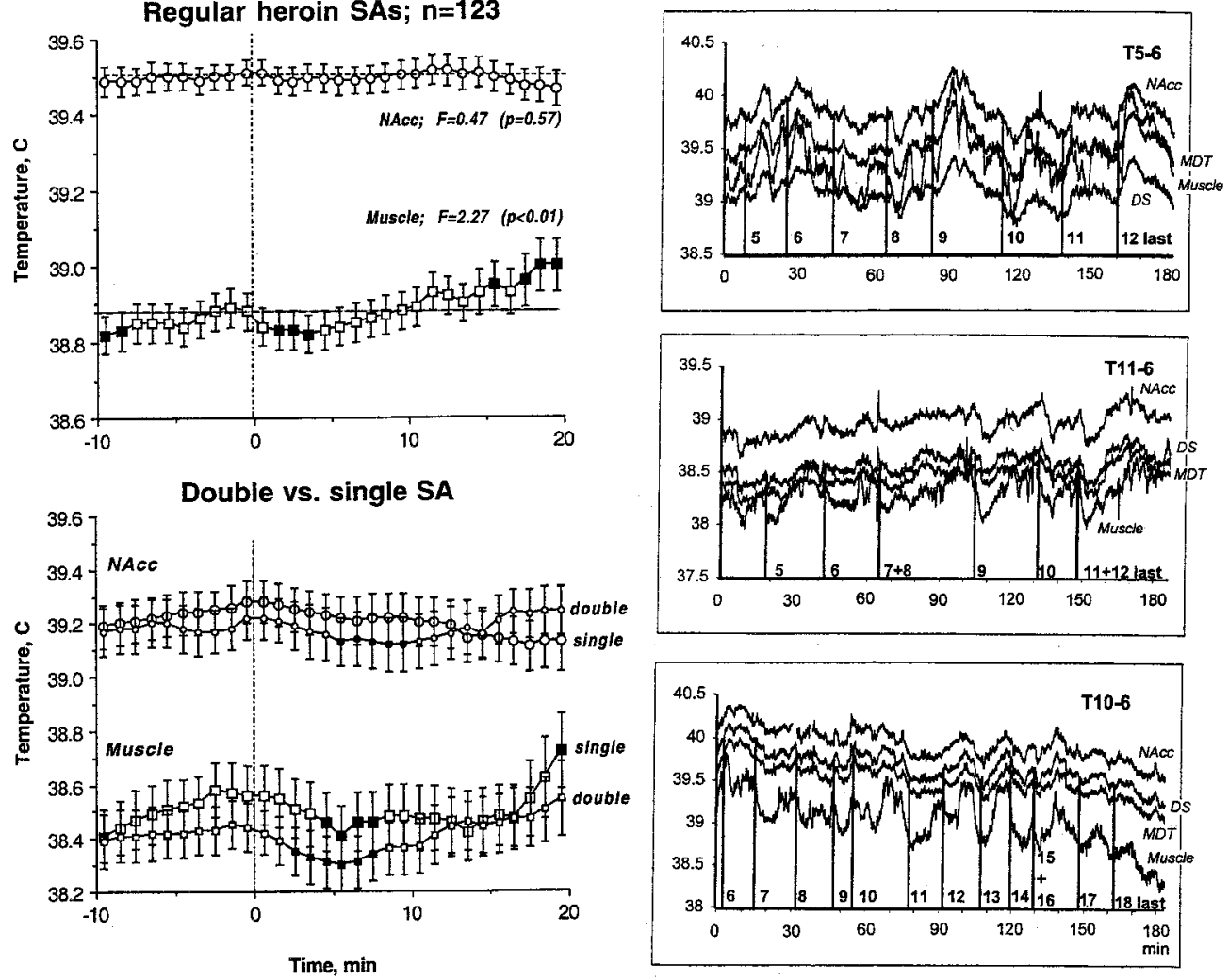

point to metabolic activation. It is important that increases in these two parameters precede the rise of body temperature, suggesting that the metabolic activation is at least partly central in origin. Brain surface temperature also increased after intraventricular injections of a selective $\mu$-agonist (Handler et al., 1992); this increase was relatively strong and long term, and it was also preceded by a short-term increase in oxygen consumption. Although evidence exists that morphine at high doses decreases cerebral blood flow in anesthetized animals (Edvinsson et al., 1993; Zamani et al., 2000) and although decreased blood flow could contribute to increases in brain temperature by restricting heat conduction back to the lungs in which the blood is cooled by air exchange, blood flow is not changed or is increased in unanesthetized animals or humans after morphine and heroin at more modest doses (Fuller and Stein, 1991; Schlaepfer et al., 1998). Thus, although generalizations from animals receiving passive injections of opiates (often at high doses and when animals are anesthetized) to animals self-administering them may be misleading, on the basis of present knowledge it appears likely that changes in neural metabolism, more than changes in blood flow or peripheral metabolism, are responsible for the brain hyperthermia seen in the present study.

The contributions of central and peripheral mechanisms underlying body hyperthermia, however, are less clear. Whereas more rapid and stronger increases in brain temperature seen in trained rats before the first heroin self-injection indicate a primary brain activation as a force behind slower and weaker body hyperhermia, changes in brain and muscle temperature during drug-taking behavior were highly correlated, and the decrease in brain temperature after naloxone injection was even slower than that in the muscle. Although this observation could argue against a central origin, it is also possible that the slower decrease in brain temperature reflects the influence of a phasic temperature eleva- tion that is consistently associated with the procedure of subcutaneous injection (Kiyatkin and Wise, 2001).

The neural mechanisms of opiate-induced metabolic activation are also not immediately clear, because opiates tend to inhibit the activity of most central neurons (Yuan et al., 1992; North, 1993). Most neuronal studies of the effects of opiates, however, were done in vitro and in anesthetized animals, and it is unclear what changes in the broad spectrum of neuronal activity occur during opiate self-administration, which is accompanied by powerful biphasic changes in general movement activity. After systemic administration of $6 \mathrm{mg} / \mathrm{kg}$ morphine, for example, presumed dopamine VTA neurons are slightly activated and nondopamine neurons are strongly inhibited (Kiyatkin, 1988). Phasic up-down fluctuations in the activity of both types of neurons are, however, consistently seen in trained rats self-administering heroin at a much smaller dose $(0.1 \mathrm{mg} / \mathrm{kg}$; Kiyatkin and Rebec, 2001). The relationships between metabolic neural activation and neuronal electric activity, moreover, may be more complex than a simple correlation. Obviously, a good deal of additional work is needed to explore the wide range of possibilities.

\section{Changes in temperature as a factor affecting neural functions}

Because most basic processes underlying neuronal excitability are temperature dependent, the $2-3^{\circ} \mathrm{C}$ increase in brain temperature occurring during heroin self-administration is sufficient to alter numerous neural functions. With a $3^{\circ} \mathrm{C}$ increase in temperature, for example, $\left[{ }^{3} \mathrm{H}\right]$ dopamine uptake assessed in embryonic mesencephalic cells doubles (Xie et al., 2000), and the amplitude of focal EPSP recorded from CA1 hippocampal cells decreases twofold (Masino and Dunwiddie, 1999). A temperature change as low as $1^{\circ} \mathrm{C}$ is sufficient to alter significantly the duration and rate 

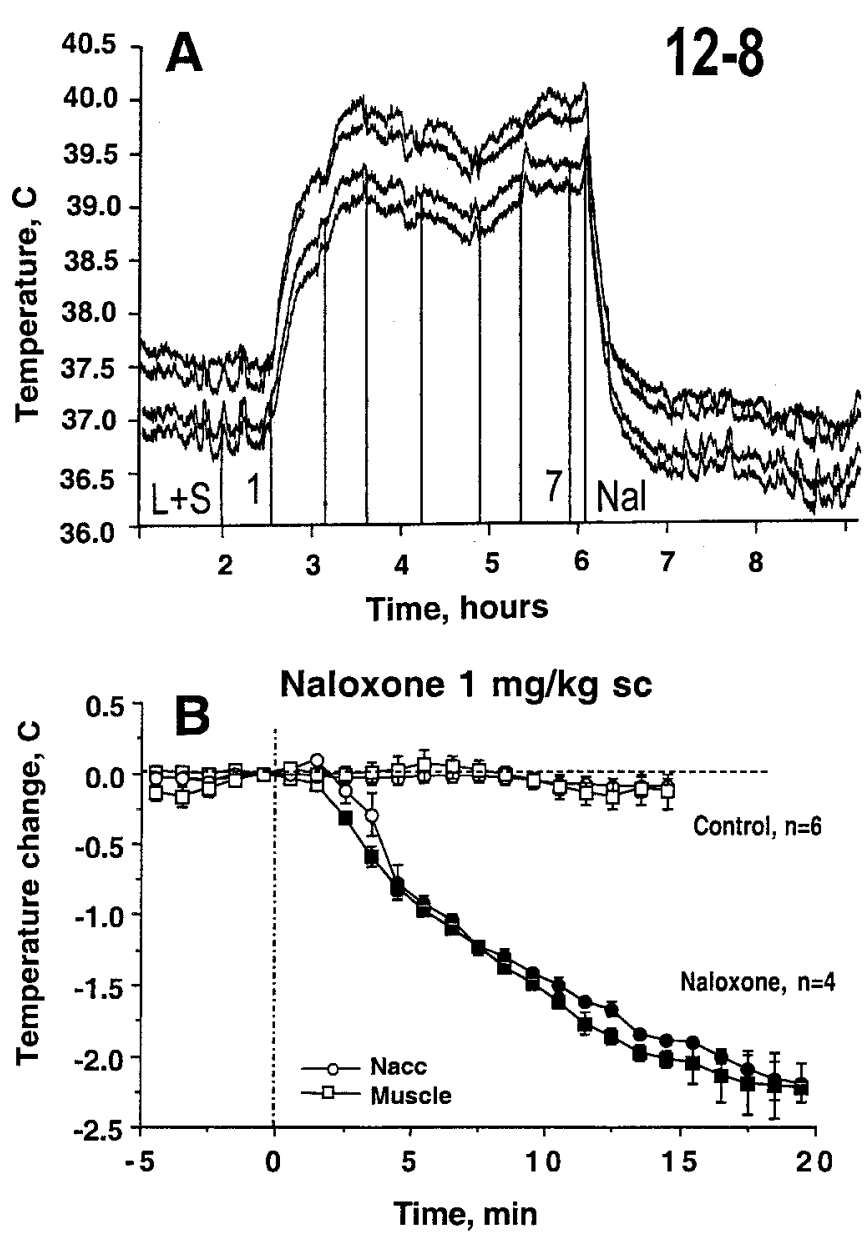

Figure 7. Mean \pm SEM changes in NAcc and muscle temperature after subcutaneous injection of naloxone during heroin self-administration session (bottom). Filled symbols are significantly lower than the last preinjection value. Both naloxone-administered and control animals made the last heroin self-injection at $-5 \mathrm{~min}$. Top shows representative example of changes in temperature induced by naloxone. $L+S$, Time interval between presentation of light-tone cue and the first heroin selfadministration of a session; Nal, naloxone.

of fall of synaptically evoked action potentials (Thompson et al., 1985), parameters that are crucial for determining neuronal discharge pattern (i.e., bursting; Thompson et al., 1985), which in turn determines neurotransmitter release (Gonon et al., 1991). Long-spike presumed dopamine VTA neurons recorded in awake, unrestrained rats, and especially during heroin selfadministration (Kiyatkin and Rebec, 1998, 2001), for example, have a shorter duration of single spikes $(\sim 3 \mathrm{msec})$ and much more dense busting than those usually reported in anesthetized animals and in vitro slices (Chiodo, 1988; Bunney et al., 1991); $2-4^{\circ} \mathrm{C}$ temperature difference between these preparations may account for these electrophysiological differences. The facts that brain temperature is significantly elevated in rats selfadministering heroin and that neural functions can be significantly altered by such elevations suggest that recording from cell cultures, tissue slices, and anesthetized animals (preparations that are usually maintained at normal body temperature or considerably below normal body temperature) may have limited utility for characterizing interactions between drug reward circuit elements in addicted animals and humans.

\section{REFERENCES}

Abrams R, Hammel HT (1964) Hypothalamic temperature in unanesthetized albino rats during feeding and sleeping. Am J Physiol 206:641-646.

Abrams R, Hammel HT (1965) Cyclic variations in hypothalamic temperature in unanesthetized rats. Am J Physiol 208:698-702.

Adler MW, Geller EB (1993) Physiological functions of opioids: temperature regulation. In: Handbook of experimental pharmacology, Vol 102/II (Herz A, ed), pp 205-238. Berlin: Springer.

Brown PL, Kiyatkin EA, Wise RA (2001) Brain hyperthermia as a reflection of emotional arousal. Soc Neurosci Abstr 27:2506.

Bullock J, Boyle J, Wang M (2001) Physiology, Ed 4. Philadelphia: Lippincott-Williams \& Wilkins.

Bunney BS, Chiodo LA, Grace AA (1991) Midbrain dopamine system electrophysiological functioning: a review and new hypothesis. Synapse 9:79-94.

Chiodo LA (1988) Dopamine-containing neurons in the mammalian central nervous system: electrophysiology and pharmacology. Neurosci Biobehav Rev 12:49-91.

Delgado JMR, Hanai T (1966) Intracerebral temperatures in freemoving cats. Am J Physiol 211:755-769.

de Wit H, Stewart J (1981) Reinstatement of cocaine-induced responding in the rat. Psychopharmacology 75:134-143.

de Wit H, Stewart J (1983) Drug reinstatement of heroin-reinforced responding in the rat. Psychopharmacology 79:29-31.

Edvinsson L, MacKenzie ET, Mc Culloch J (1993) Cerebral blood flow and metabolism. New York: Raven.

Erickson CA, Jung MW, McNaughton BL, Barnes CA (1996) Contribution of single-unit spike waveform changes to temperature-induced alterations in hippocampal population spikes. Exp Brain Res 107:348-360.

Fuller SA, Stein EA (1991) Effects of heroin and naloxone on cerebral blood flow in the conscious rat. Pharmacol Biochem Behav 40:339-444.

Gonon F, Suaud-Chagney MF, Mermet CC, Buda M (1991) Relationships between impulse flow and extracellular catecholamine levels as studied by in vivo electrochemistry in CNS. In: Volume transmission in the brain (Fuxe K, Agnati LF, eds), pp 337-350. New York: Raven.

Grimm JW, Hope BT, Wise RA, Shaham Y (2001) Neuroadaptation incubation of cocaine craving after withdrawal. Nature 412:141-142.

Handler CM, Geller EB, Adler MW (1992) Effects of $\mu-$, k-, and $\delta$-selective opioid agonists on thermoregulation in the rat. Pharmacol Biochem Behav 43:1209-1216.

Kiyatkin EA (1988) Morphine-induced modification of the functional properties of ventral tegmental neurons in conscious rats. Int J Neurosci 41:57-70.

Kiyatkin EA, Rebec GV (1998) Heterogeneity of ventral tegmental area neurons: single unit recording and iontophoresisa in awake, unrestrained rats. Neuroscience 85:1285-1309.

Kiyatkin EA, Rebec GV (2001) Impulse activity of ventral tegmental area neurons during heroin self-administration in rats. Neuroscience 102:565-580.

Kiyatkin EA, Wise RA (2001) Striatal hyperthermia associated with arousal: intracranial thermorecordings in behaving rats. Brain Res 918:141-152.

Kiyatkin EA, Stein EA (1993) Behavior-associated changes in blood pressure during heroin self-administration. Pharmacol Biochem Behav 46:561-567.

Lynch TJ, Martinez RP, Furman MB, Geller EB, Adler MW (1987) A calorimetric analysis of body temperature changes produced by morphine, metadone, and U50,488H. In: Problems of drug dependence 1986. NIDA Res Monograph 76:82. Rockville, MD: National Institute on Drug Abuse.

Lynch TJ, Adler MW, Eisenstein TK (1990) Comparison of the mechanisms of interleukin-1 and morphine-induced hyperthermia in the rat. Ann NY Acad Sci 594:469-471.

Masino SA, Dunwiddie TV (1999) Temperature-dependent modulation of excitatory transmission in hippocampal slices is mediated by extracellular adenosine. J Neurosci 19:1932-1939.

Meil WM, See RE (1996) Conditioned cue recovery of responding following withdrawal from self-administered cocaine in rats: an animal model of relapse. Behav Pharmacol 7:754-763.

Moser E, Mathesen I, Andersen P (1993) Association between brain temperature and dentate field potentials in exploring and swimming rats. Science 259:1324-1326.

North RA (1993) Opioid action on membrane ion channels. In: Handbook of experimental pharmacology, Vol 102/I (Herz A, ed), pp 773797. Berlin: Springer.

Pavlov IP (1927) Conditioned reflexes. Oxford: Oxford UP.

Paxinos G, Watson C (1986) The rat brain in stereotaxic coordinates. Sydney: Academic.

Robinson TE, Berridge KC (2001) Incentive-sensitization, addiction. Addiction 96:103-114 
Rosen AD (2001) Nonlinear temperature modulation of sodium channel kinetics in GH3 cells. Biochim Biophys Acta 1511:391-396.

Satinoff E (1978) Neural organization and evolution of thermal regulation in mammals. Science 201:16-22.

Schlaepfer TE, Strain EC, Greenberg BD, Preston KL, Lancaster E, Bigelow GE, Barta PE, Pearlson GD (1998) Site of opioid action in the human brain: mu and kappa agonists' subjective and cerebral blood flow effects. Am J Psychiatry 155:470-473.

Schultz W (1997) A neural substrate of prediction and reward. Science 275:1593-1599.

Schultz W, Dickinson A (2000) Neuronal coding of prediction errors. Annu Rev Neurosci 23:473-500.
Thompson SM, Musakawa LM, Rince DA (1985) Temperature dependence of intrinsic membrane properties and synaptic potentials in hippocampal CA1 neurons in vitro. J Neurosci 5:817-824.

Xie T, McGann UD, Kim S, Yuan J, Ricaurte GA (2000) Effect of temperature on dopamine transporter function and intracellular accumulation of methamphetamine: implications for methamphetamineinduced dopaminergic neurotoxicity. J Neurosci 20:7838-7845.

Yuan XR, Madamba S, Siggins GR (1992) Opioid peptides reduce synaptic transmission in the nucleus accumbens. Neurosci Lett 134:223-228.

Zamani R, Semnanian S, Fethollahi Y, Hajizadeh S (2000) Systemic naloxone enhances cerebral blood flow in anesthetized morphinedependent rats. Eur J Pharmacol 408:299-304. 